\title{
Free trees and the optimal bound in Wehrung's theorem
}

\author{
by
}

\author{
Pavel Růžička (Praha)
}

\begin{abstract}
We prove that there is a distributive $(\vee, 0,1)$-semilattice $\mathcal{G}$ of size $\aleph_{2}$ such that there is no weakly distributive $(\vee, 0)$-homomorphism from $\operatorname{Con}_{c} A$ to $\mathcal{G}$ with 1 in its range, for any algebra $A$ with either a congruence-compatible structure of a $(\vee, 1)$-semilattice or a congruence-compatible structure of a lattice. In particular, $\mathcal{G}$ is not isomorphic to the $(\vee, 0)$-semilattice of compact congruences of any lattice. This improves Wehrung's solution of Dilworth's Congruence Lattice Problem, by giving the best cardinality bound possible. The main ingredient of our proof is the modification of Kuratowski's Free Set Theorem, which involves what we call free trees.
\end{abstract}

1. Introduction. Congruence lattices of universal algebras correspond to algebraic lattices. By the theorem of N. Funayama and T. Nakayama [2], the congruence lattice of a lattice is, in addition, distributive (see also [3, II. 3. Theorem 11]). On the other hand, R. P. Dilworth proved that every finite distributive lattice is isomorphic to the congruence lattice of a finite lattice (first published in [5]) and he conjectured that every distributive algebraic lattice is isomorphic to the congruence lattice of a lattice (see again [5]). This conjecture, referred to as the Congruence Lattice Problem, despite many attempts (see surveys [3, Appendix C] and [12]), remained open for over sixty years until, recently, F. Wehrung disproved it in [17].

Wehrung's solution involves a combination of new ideas (see, in particular, Lemmas 4.4, 5.1, and 6.2 in [17]) and methods developed in earlier papers, which originated in [14] and were pursued further in $[9,10,11,13,15]$. In these papers, counterexamples to various problems related to the Congruence Lattice Problem were obtained. The optimal cardinality bound for all these counterexamples is $\aleph_{2}$, but Wehrung's argument requires an algebraic distributive lattice with at least $\aleph_{\omega+1}$ compact elements. In the present

2000 Mathematics Subject Classification: 06B15, 06B10, 06A12, 08A30, 08B10.

Key words and phrases: lattice, algebraic, semilattice, distributive, congruence, weakly distributive, free tree.

This work is part of the research project MSM 0021620839, financed by MSMT; it is also partly supported by INTAS project 03-51-4110 and grant GAUK 448/2004/B-MAT. 
paper, we improve Wehrung's result by proving that there is a counterexample of size $\aleph_{2}$. As in the related cases, $\aleph_{2}$ turns out to be the optimal cardinality bound for a negative solution of the Congruence Lattice Problem. Our proof closely follows Wehrung's ideas. The main difference consists in an enhancement of Kuratowski's Free Set Theorem by a new combinatorial principle which involves finite trees.

Wehrung's construction in [17] uses a "free" distributive extension of a $(\vee, 0)$-semilattice, a functor that assigns to every $(\vee, 0)$-semilattice a distributive $(\vee, 0)$-semilattice, constructed previously by M. Ploščica and J. Tůma in [9]. The main features of this construction for the refutation of the Congruence Lattice Problem are extracted in the so-called Evaporation Lemma [17, Lemma 4.4]. We generalize this idea by defining a diluting functor whose properties are sufficient to prove the Evaporation Lemma, and we prove that the free distributive extension of a $(\vee, 0)$-semilattice is, indeed, a diluting functor.

Further, we modify Kuratowski's Free Set Theorem, the combinatorial essence of the above mentioned counterexamples. Given a set $\Omega$ and a map $\Phi:[\Omega]^{<\omega} \rightarrow[\Omega]^{<\omega}$, we define a free $k$-tree (with respect to $\Phi$ ), for every positive integer $k$, which is a $k$-ary tree with some combinatorial properties derived from Kuratowski's Free Set Theorem. We prove that a free $k$-tree exists whenever the cardinality of the set $\Omega$ is at least $\aleph_{k-1}$, and we apply the existence of a free 3 -tree in every set of cardinality at least $\aleph_{2}$ to attain the optimal cardinality bound in the Wehrung's result.

2. Basic concepts. A $(\vee, 0)$-semilattice $S$ is distributive if for any $\boldsymbol{a}, \boldsymbol{b}, \boldsymbol{c} \in S$ satisfying $\boldsymbol{c} \leq \boldsymbol{a} \vee \boldsymbol{b}$, there are $\boldsymbol{a}^{\prime} \leq \boldsymbol{a}$ and $\boldsymbol{b}^{\prime} \leq \boldsymbol{b}$ such that $\boldsymbol{a}^{\prime} \vee \boldsymbol{b}^{\prime}=\boldsymbol{c}$. A homomorphism $\mu: S \rightarrow T$ of join-semilattices is called weakly distributive at $\boldsymbol{x} \in S$ if for all $\boldsymbol{y}_{0}, \boldsymbol{y}_{1} \in T$ such that $\mu(\boldsymbol{x}) \leq \boldsymbol{y}_{0} \vee \boldsymbol{y}_{1}$, there are $\boldsymbol{x}_{0}, \boldsymbol{x}_{1} \in S$ such that $\boldsymbol{x} \leq \boldsymbol{x}_{0} \vee \boldsymbol{x}_{1}$ and $\mu\left(\boldsymbol{x}_{i}\right) \leq \boldsymbol{y}_{i}$ for all $i<2$ (see [17]). The homomorphism $\mu$ is weakly distributive if it is weakly distributive at every element of $S$.

Let $A$ be an algebra. We say that an $n$-ary operation $f$ on $A$ is congruence-compatible (see $[8,17]$ ) if for every congruence $\theta$ of $A,\left(x_{i}, y_{i}\right) \in \theta$ for $i=0, \ldots, n-1$ implies that $\left(f\left(x_{0}, \ldots, x_{n-1}\right), f\left(y_{0}, \ldots, y_{n-1}\right)\right) \in \theta$. In particular, a semilattice operation $\vee$, resp. $\wedge$, on $A$ is congruence-compatible providing that $(x, y) \in \theta$ implies $(x \vee z, y \vee z) \in \theta$, resp. $(x \wedge z, y \wedge z) \in \theta$, for all $x, y, z \in A$ and $\theta \in \operatorname{Con} A$.

Given an algebra $A$ and elements $x, y \in A$, we denote by $\Theta_{A}(x, y)$ the smallest congruence (i.e., intersection of all the congruences) of $A$ identifying $x$ and $y$. We denote by $\operatorname{Con} A$, resp. $\operatorname{Con}_{c} A$ the lattice of all congruences of $A$, resp. the join-semilattice of all compact congruences of $A$. We say that $A$ has permutable congruences if $\boldsymbol{a} \vee \boldsymbol{b}=\boldsymbol{a} \circ \boldsymbol{b}=\boldsymbol{b} \circ \boldsymbol{a}$ for all $\boldsymbol{a}, \boldsymbol{b} \in \operatorname{Con} A$. 
We will use the standard set-theoretic notation and terminology. We identify each ordinal number with the set of its predecessors, in particular, $n=\{0, \ldots, n-1\}$ for each positive integer $n$. We denote by $\omega$ the first infinite ordinal, and by $\omega_{n}$ the first ordinal of size $\aleph_{n}$, for every positive integer $n$. For a set $X$, we denote by $[X]^{<\omega}$ the set of all finite subsets of $X$ and by $[X]^{n}$ the set of all its $n$-element subset, for every natural number $n$. We denote by $|X|$ the cardinality of a set $X$. As in [17], we put $\varepsilon(n)=n \bmod 2$ for every integer $n$.

3. Diluting functors. Denote by $\mathbf{S}$ the category of $(\vee, 0)$-semilattices (with ( $\vee, 0)$-homomorphisms).

Definition 1. An expanding functor on $\mathbf{S}$ is a pair $(\mathcal{F}, \iota)$, where $\mathcal{F}$ is an endofunctor on $\mathbf{S}$ and $\iota$ is a natural transformation from the identity to $\mathcal{F}$ such that $\iota_{S}: S \rightarrow \mathcal{F}(S)$ is an embedding for every $(\vee, 0)$-semilattice $S$. We shall denote the expanding functor above by $\mathcal{F}$ once the natural transformation $\iota$ is understood, and we shall identify $\iota_{S}(\boldsymbol{x})$ with $\boldsymbol{x}$ for all $\boldsymbol{x} \in S$.

An expanding functor $\mathcal{F}$ on $\mathbf{S}$ is a diluting functor if for all $(\vee, 0)$-semilattices $S$ and $T$ and every $(\vee, 0)$-homomorphism $f: S \rightarrow T$, the following property is satisfied: for all $\boldsymbol{v} \in \mathcal{F}(S)$ and $\boldsymbol{u}_{0}, \boldsymbol{u}_{1} \in \mathcal{F}(T)$, if $\mathcal{F}(f)(\boldsymbol{v}) \leq$ $\boldsymbol{u}_{0} \vee \boldsymbol{u}_{1}$, then there are $\boldsymbol{x}_{0}, \boldsymbol{x}_{1} \in \mathcal{F}(S)$ and $\boldsymbol{y} \in S$ such that

$$
f(\boldsymbol{y}) \leq \boldsymbol{u}_{0} \vee \boldsymbol{u}_{1}, \quad \mathcal{F}(f)\left(\boldsymbol{x}_{i}\right) \leq \boldsymbol{u}_{i} \quad \text { for all } i<2, \quad \boldsymbol{v} \leq \boldsymbol{x}_{0} \vee \boldsymbol{x}_{1} \vee \boldsymbol{y} .
$$

Given a $(\vee, 0)$-semilattice $S$ and subsets $U, V$ of $S$, we shall use the notation

$$
U \vee V=\{\boldsymbol{u} \vee \boldsymbol{v} \mid \boldsymbol{u} \in U \text { and } \boldsymbol{v} \in V\} .
$$

Lemma 3.1. Let $\mathcal{F}$ be an expanding functor on $\mathbf{S}$. Let $S$ be a $(\vee, 0)$-semilattice and let $S_{i}, i<2$, be $(\vee, 0)$-subsemilattices of $S$ such that $S=S_{0} \vee S_{1}$ and there are retractions $r_{i}: S \rightarrow S_{i}$ for $i<2$. Put $s_{i}=\mathcal{F}\left(r_{i}\right)$ for every $i<2$. Let $\boldsymbol{u}_{i} \in \mathcal{F}\left(S_{i}\right), i<2$, be such that $s_{i}\left(\boldsymbol{u}_{1-i}\right)=0$ for all $i<2$. Then for every $\boldsymbol{y} \in S$ such that $\boldsymbol{y} \leq \boldsymbol{u}_{0} \vee \boldsymbol{u}_{1}$, there are $\boldsymbol{y}_{i} \in S_{i}, i<2$, such that $\boldsymbol{y} \leq \boldsymbol{y}_{0} \vee \boldsymbol{y}_{1}$ and $\boldsymbol{y}_{i} \leq \boldsymbol{u}_{i}$ for all $i<2$.

Proof. Put $\boldsymbol{y}_{i}=r_{i}(\boldsymbol{y})$ for every $i<2$. Since $S=S_{0} \vee S_{1}$, there are $\boldsymbol{y}_{i}^{\prime} \in S_{i}$ for $i<2$ such that $\boldsymbol{y}=\boldsymbol{y}_{0}^{\prime} \vee \boldsymbol{y}_{1}^{\prime}$. Since the maps $r_{i}, i<2$, are retractions, $\boldsymbol{y}_{i}^{\prime}=r_{i}\left(\boldsymbol{y}_{i}^{\prime}\right) \leq r_{i}\left(\boldsymbol{y}_{i}\right)=\boldsymbol{y}_{i}$ for all $i<2$, whence $\boldsymbol{y} \leq \boldsymbol{y}_{0} \vee \boldsymbol{y}_{1}$.

It remains to prove that $\boldsymbol{y}_{i} \leq \boldsymbol{u}_{i}$ for all $i<2$. Fix $i<2$. Since $s_{i} \uparrow S=r_{i}$ and $s_{i}: \mathcal{F}(S) \rightarrow \mathcal{F}\left(S_{i}\right)$ is a retraction, $s_{i}\left(\boldsymbol{u}_{i}\right)=\boldsymbol{u}_{i}$. Since, by the assumptions, $s_{i}\left(\boldsymbol{u}_{1-i}\right)=0$, we conclude that

$$
\boldsymbol{y}_{i}=s_{i}(\boldsymbol{y}) \leq s_{i}\left(\boldsymbol{u}_{0} \vee \boldsymbol{u}_{1}\right)=s_{i}\left(\boldsymbol{u}_{0}\right) \vee s_{i}\left(\boldsymbol{u}_{1}\right)=s_{i}\left(\boldsymbol{u}_{i}\right)=\boldsymbol{u}_{i} .
$$

Given an expanding functor $\mathcal{F}$, define $\mathcal{F}^{0}$ to be the identity functor and, inductively, $\mathcal{F}^{n+1}=\mathcal{F} \circ \mathcal{F}^{n}$ for every natural number $n$. By our assumption, the inclusion map defines a natural transformation from the identity functor 
on $\mathbf{S}$ to $\mathcal{F}$, therefore we can define $\mathcal{F}^{\infty}(S)=\bigcup_{n \in \omega} \mathcal{F}^{n}(S)$, resp. $\mathcal{F}^{\infty}(f)=$ $\bigcup_{n \in \omega} \mathcal{F}^{n}(f)$, for every $(\vee, 0)$-semilattice $S$, resp. every $(\vee, 0)$-homomorphism $f: S \rightarrow T$, and again the inclusion map defines a natural transformation from the identity functor on $\mathbf{S}$ to $\mathcal{F}^{\infty}$. In particular, if $\mathcal{F}$ is an expanding functor on $\mathbf{S}$, then $\mathcal{F}^{\infty}$ is expanding as well.

Lemma 3.2. Let $\mathcal{F}$ be a diluting functor on $\mathbf{S}$. Then the functor $\mathcal{F}^{\infty}$ is diluting as well.

Proof. Let $S$ and $T$ be $(\vee, 0)$-semilattices and let $f: S \rightarrow T$ be a $(\vee, 0)$ homomorphism. Let $\boldsymbol{v} \in \mathcal{F}^{\infty}(S)$ and let $\boldsymbol{u}_{0}, \boldsymbol{u}_{1} \in \mathcal{F}^{\infty}(T)$ be such that $\mathcal{F}^{\infty}(f)(\boldsymbol{v}) \leq \boldsymbol{u}_{0} \vee \boldsymbol{u}_{1}$. We are looking for $\boldsymbol{x}_{0}, \boldsymbol{x}_{1} \in \mathcal{F}^{\infty}(S)$ and $\boldsymbol{y} \in S$ such that

$$
f(\boldsymbol{y}) \leq \boldsymbol{u}_{0} \vee \boldsymbol{u}_{1}, \quad \mathcal{F}^{\infty}(f)\left(\boldsymbol{x}_{i}\right) \leq \boldsymbol{u}_{i} \quad \text { for all } i<2, \quad \boldsymbol{v} \leq \boldsymbol{x}_{0} \vee \boldsymbol{x}_{1} \vee \boldsymbol{y} .
$$

We shall argue by induction on the least natural number $n$ such that $\boldsymbol{v} \in$ $\mathcal{F}^{n}(S)$. If $n=0$, we put $\boldsymbol{x}_{0}=\boldsymbol{x}_{1}=0, \boldsymbol{y}=\boldsymbol{v}$, and we are done. Suppose that $\boldsymbol{v} \in \mathcal{F}^{n+1}(S)$ for some natural number $n$, and that the property is proved at stage $n$. Let $k \geq n$ be a natural number such that $\boldsymbol{u}_{0}, \boldsymbol{u}_{1} \in \mathcal{F}^{k+1}(T)$. Denote by $g$ the composition of the $(\vee, 0)$-homomorphism $\mathcal{F}^{n}(f)$ and the inclusion map from $\mathcal{F}^{n}(T)$ to $\mathcal{F}^{k}(T)$. By applying the assumption that $\mathcal{F}$ is a diluting functor to the $(\vee, 0)$-homomorphism $g: \mathscr{F}^{n}(S) \rightarrow \mathcal{F}^{k}(T)$, we obtain elements $\boldsymbol{x}_{0}^{\prime}, \boldsymbol{x}_{1}^{\prime} \in \mathcal{F}^{n+1}(S)$ and $\boldsymbol{y}^{\prime} \in \mathcal{F}^{n}(S)$ such that

$$
g\left(\boldsymbol{y}^{\prime}\right) \leq \boldsymbol{u}_{0} \vee \boldsymbol{u}_{1}, \quad \mathcal{F}(g)\left(\boldsymbol{x}_{i}^{\prime}\right) \leq \boldsymbol{u}_{i} \quad \text { for all } i<2, \quad \boldsymbol{v} \leq \boldsymbol{x}_{0}^{\prime} \vee \boldsymbol{x}_{1}^{\prime} \vee \boldsymbol{y}^{\prime} .
$$

Since $g\left(\boldsymbol{y}^{\prime}\right) \leq \boldsymbol{u}_{0} \vee \boldsymbol{u}_{1}$ implies $\mathcal{F}^{\infty}(f)\left(\boldsymbol{y}^{\prime}\right) \leq \boldsymbol{u}_{0} \vee \boldsymbol{u}_{1}$, there are, by the induction hypothesis, elements $\boldsymbol{x}_{0}^{\prime \prime}, \boldsymbol{x}_{1}^{\prime \prime} \in \mathcal{F}^{\infty}(S)$ and $\boldsymbol{y} \in S$ such that

$$
f(\boldsymbol{y}) \leq \boldsymbol{u}_{0} \vee \boldsymbol{u}_{1}, \quad \mathcal{F}^{\infty}(f)\left(\boldsymbol{x}_{i}^{\prime \prime}\right) \leq \boldsymbol{u}_{i} \quad \text { for all } i<2, \quad \boldsymbol{y}^{\prime} \leq \boldsymbol{x}_{0}^{\prime \prime} \vee \boldsymbol{x}_{1}^{\prime \prime} \vee \boldsymbol{y} .
$$

Now it is easy to conclude that $\boldsymbol{x}_{i}=\boldsymbol{x}_{i}^{\prime} \vee \boldsymbol{x}_{i}^{\prime \prime}$ for $i<2$ and $\boldsymbol{y}$ are the desired elements.

As in [17], denote by $\mathcal{L}$ the functor from the category of sets to $\mathbf{S}$ which assigns to a set $\Omega$ the $(\vee, 0,1)$-semilattice $\mathcal{L}(\Omega)$ defined by generators 1 and $\boldsymbol{a}_{0}^{\xi}, \boldsymbol{a}_{1}^{\xi}$ for $\xi \in \Omega$, subjected to the relations

$$
\boldsymbol{a}_{0}^{\xi} \vee \boldsymbol{a}_{1}^{\xi}=1 \text { for all } \xi \in \Omega,
$$

and which assigns to a map $f: X \rightarrow Y$ the unique $(\vee, 0,1)$-homomorphism $\mathcal{L}(f): \mathcal{L}(X) \rightarrow \mathcal{L}(Y)$ such that $\mathcal{L}(f)\left(\boldsymbol{a}_{i}^{\xi}\right)=\boldsymbol{a}_{i}^{f(\xi)}$ for all $\xi \in X$ and all $i<2$.

Given a finite subset $A$ of $\Omega$ and a map $\varphi: A \rightarrow 2$, we put $\boldsymbol{a}_{\varphi}^{A}=$ $\bigvee_{\alpha \in A} \boldsymbol{a}_{\varphi(\alpha)}^{\alpha}$. By Corollary 4.2 below, the following lemma is a generalization of Wehrung's original "Evaporation Lemma" [17, Lemma 4.4].

Lemma 3.3. Let $\mathcal{F}$ be a diluting functor on $\mathbf{S}$. Define $\mathcal{G}=\mathcal{F} \circ \mathcal{L}$. Let $\Omega$ be a set, let $A_{0}, A_{1}$ be finite disjoint subsets of $\Omega$, and let $\delta \in \Omega \backslash\left(A_{0} \cup A_{1}\right)$. 
Let $\boldsymbol{v} \in \mathcal{G}(\Omega \backslash\{\delta\})$, let $\varphi_{i}: A_{i} \rightarrow 2$, and let $\boldsymbol{u}_{i} \in \mathcal{G}\left(\Omega \backslash A_{1-i}\right)$ for $i<2$. Then

$$
\boldsymbol{v} \leq \boldsymbol{u}_{0} \vee \boldsymbol{u}_{1} \quad \text { and } \quad \boldsymbol{u}_{i} \leq \boldsymbol{a}_{\varphi_{i}}^{A_{i}}, \boldsymbol{a}_{i}^{\delta} \quad \text { for all } i<2
$$

implies that $\boldsymbol{v}=0$.

Proof. Denote by $f$ the inclusion map from $\Omega \backslash\{\delta\}$ to $\Omega$, and observe that $\mathcal{L}(f)$ corresponds to the inclusion $\mathcal{L}(\Omega \backslash\{\delta\}) \subseteq \mathcal{L}(\Omega)$. Since $\mathcal{F}$ is diluting, there are elements $\boldsymbol{x}_{0}, \boldsymbol{x}_{1} \in \mathcal{G}(\Omega \backslash\{\delta\})$ and $\boldsymbol{y} \in \mathcal{L}(\Omega \backslash\{\delta\})$ such that

$$
\boldsymbol{y} \leq \boldsymbol{u}_{0} \vee \boldsymbol{u}_{1}, \quad \mathcal{G}(f)\left(\boldsymbol{x}_{i}\right) \leq \boldsymbol{u}_{i} \quad \text { for all } i<2, \quad \boldsymbol{v} \leq \boldsymbol{x}_{0} \vee \boldsymbol{x}_{1} \vee \boldsymbol{y} .
$$

Fix $i<2$. There is a unique retraction $p_{i}: \mathcal{L}(\Omega) \rightarrow \mathcal{L}(\Omega \backslash\{\delta\})$ satisfying $p_{i}\left(\boldsymbol{a}_{i}^{\delta}\right)=0$ and $p_{i}\left(\alpha_{1-i}^{\delta}\right)=1$. Observe that $q_{i}=\mathcal{F}\left(p_{i}\right): \mathcal{G}(\Omega) \rightarrow \mathcal{G}(\Omega \backslash$ $\{\delta\})$ is a retraction with respect to $\mathcal{G}(f)$. Since $\boldsymbol{x}_{i} \in \mathcal{G}(\Omega \backslash\{\delta\})$ we have $q_{i}\left(\mathcal{G}(f)\left(\boldsymbol{x}_{i}\right)\right)=\boldsymbol{x}_{i}$, while, by our assumptions, $q_{i}\left(\boldsymbol{a}_{i}^{\delta}\right)=0$. Since $\mathcal{G}(f)\left(\boldsymbol{x}_{i}\right) \leq$ $\boldsymbol{u}_{i} \leq \boldsymbol{a}_{i}^{\delta}$, we conclude that $\boldsymbol{x}_{i}=0$.

Let $r_{i}: \mathcal{L}(\Omega) \rightarrow \mathcal{L}\left(\Omega \backslash A_{1-i}\right)$ be a unique retraction such that $r_{i}\left(\boldsymbol{a}_{\varphi_{1-i}}^{A_{1-i}}\right)$ $=0$, and put $s_{i}=\mathcal{F}\left(r_{i}\right)$. From $\boldsymbol{u}_{1-i} \leq \boldsymbol{a}_{\varphi_{1-i}}^{A_{1-i}}$ it follows that $s_{i}\left(\boldsymbol{u}_{1-i}\right)=0$. By Lemma 3.1, there are $\boldsymbol{y}_{j} \in \mathcal{L}\left(\Omega \backslash A_{1-j}\right)$ with $\boldsymbol{y}_{j} \leq \boldsymbol{u}_{j}$ for all $j<2$ such that $\boldsymbol{y} \leq \boldsymbol{y}_{0} \vee \boldsymbol{y}_{1}$. Since $\boldsymbol{y}_{j} \leq \boldsymbol{u}_{j} \leq \boldsymbol{a}_{\varphi_{j}}^{A_{j}}, \boldsymbol{a}_{j}^{\delta}$ and $\delta \notin A_{j}$, we conclude that $\boldsymbol{y}_{j}=0$ for all $j<2$.

4. Free distributive extension is diluting. We summarize the main properties of the construction of the extension $\mathcal{R}(S)$ of a $(\vee, 0)$-semilattice $S$ (see [9, Section 2]) referring to the outline in [17, Sections 3,4]. We shall prove that the functor $\mathcal{R}$ is diluting. For a $(\vee, 0)$-semilattice $S$, we shall put $\mathcal{C}(S)=\left\{(\boldsymbol{a}, \boldsymbol{b}, \boldsymbol{c}) \in S^{3} \mid \boldsymbol{c} \leq \boldsymbol{a} \vee \boldsymbol{b}\right\}$. We say that a finite subset $\boldsymbol{v}$ of $\mathcal{C}(S)$ is reduced if the following properties are satisfied:

(1) the set $\boldsymbol{v}$ contains exactly one triple of the form $(\boldsymbol{a}, \boldsymbol{a}, \boldsymbol{a})$; we define $\pi(\boldsymbol{v})=\boldsymbol{a}$ and $\boldsymbol{v}^{*}=\boldsymbol{v} \backslash\{(\boldsymbol{a}, \boldsymbol{a}, \boldsymbol{a})\}$.

(2) $(\boldsymbol{a}, \boldsymbol{b}, \boldsymbol{c}) \in \boldsymbol{v}$ and $(\boldsymbol{b}, \boldsymbol{a}, \boldsymbol{c}) \in \boldsymbol{v}$ implies that $\boldsymbol{a}=\boldsymbol{b}=\boldsymbol{c}$, for all $\boldsymbol{a}, \boldsymbol{b}, \boldsymbol{c} \in S$.

(3) if $(\boldsymbol{a}, \boldsymbol{b}, \boldsymbol{c}) \in \boldsymbol{v}^{*}$, then $\boldsymbol{a}, \boldsymbol{b}, \boldsymbol{c} \not \leq \pi(\boldsymbol{v})$, for all $\boldsymbol{a}, \boldsymbol{b}, \boldsymbol{c} \in S$.

Observe that if $\boldsymbol{v}$ is a reduced subset of $\mathcal{C}(S)$ and $\boldsymbol{u} \subseteq \boldsymbol{v}^{*}$, then $\boldsymbol{u} \cup\{(0,0,0)\}$ is a reduced subset as well.

We denote by $\mathcal{R}(S)$ the set of all reduced subsets of $\mathcal{C}(S)$. By [9, Lemma 2.1] (see also [17, Corollary 3.2]), $\mathcal{R}(S)$ is a $(\vee, 0)$-semilattice with respect to the partial ordering $\leq$ defined by

$$
\boldsymbol{v} \leq \boldsymbol{u} \quad \text { iff } \quad \text { for all }(\boldsymbol{a}, \boldsymbol{b}, \boldsymbol{c}) \in \boldsymbol{v} \backslash \boldsymbol{u} \text { either } \boldsymbol{a} \leq \pi(\boldsymbol{u}) \text { or } \boldsymbol{c} \leq \pi(\boldsymbol{u}),
$$
and the assignment $\boldsymbol{v} \mapsto\{(\boldsymbol{v}, \boldsymbol{v}, \boldsymbol{v})\}$ is a $(\vee, 0)$-embedding from $S$ into $\mathcal{R}(S)$. 
As in [17], we use the symbol $\bowtie_{S}$ to denote the elements of $\mathcal{R}(S)$ defined as

$$
\bowtie_{S}(\boldsymbol{a}, \boldsymbol{b}, \boldsymbol{c})= \begin{cases}\boldsymbol{c} & \text { if either } \boldsymbol{a}=\boldsymbol{b} \text { or } \boldsymbol{b}=0 \text { or } \boldsymbol{c}=0, \\ 0 & \text { if } \boldsymbol{a}=0, \\ \{(0,0,0),(\boldsymbol{a}, \boldsymbol{b}, \boldsymbol{c})\} & \text { otherwise, }\end{cases}
$$

for all $(\boldsymbol{a}, \boldsymbol{b}, \boldsymbol{c}) \in \mathcal{C}(S)$. Recall that by formula (3.3) in [17],

$$
\boldsymbol{x}=\bigvee\left\{\bowtie_{S}(\boldsymbol{a}, \boldsymbol{b}, \boldsymbol{c}) \mid(\boldsymbol{a}, \boldsymbol{b}, \boldsymbol{c}) \in \boldsymbol{x}\right\} \quad \text { for all } \boldsymbol{x} \in \mathcal{R}(S) .
$$

By [17, Proposition 3.5], every $(\vee, 0)$-homomorphism $f: S \rightarrow T$ extends to a unique $(\vee, 0)$-homomorphism $\mathcal{R}(f): S \rightarrow T$ such that

$$
\mathcal{R}(f)\left(\bowtie_{S}(\boldsymbol{a}, \boldsymbol{b}, \boldsymbol{c})\right)=\bowtie_{T}(f(\boldsymbol{a}), f(\boldsymbol{b}), f(\boldsymbol{c})) \quad \text { for all }(\boldsymbol{a}, \boldsymbol{b}, \boldsymbol{c}) \in \mathcal{C}(S),
$$

and the assignment $S \mapsto \mathcal{R}(S), f \mapsto \mathcal{R}(f)$, is a functor on the category $\mathbf{S}$. It follows that if $f: S \rightarrow T$ is a $(\vee, 0)$-homomorphism, $\boldsymbol{v} \in S$, and $\boldsymbol{u} \in T$, then

$$
\mathcal{R}(f)(\boldsymbol{v}) \leq \boldsymbol{u} \quad \text { iff } \quad \bowtie_{T}(f(\boldsymbol{a}), f(\boldsymbol{b}), f(\boldsymbol{c})) \leq \boldsymbol{u} \quad \text { for all }(\boldsymbol{a}, \boldsymbol{b}, \boldsymbol{c}) \in \boldsymbol{v} .
$$

Lemma 4.1. The functor $\mathcal{R}$ is diluting.

Proof. Let $S$ and $T$ be $(\vee, 0)$-semilattices and let $f: S \rightarrow T$ be a $(\vee, 0)$ homomorphism. We have to verify that for every $\boldsymbol{v} \in \mathcal{R}(S)$ and $\boldsymbol{u}_{0}, \boldsymbol{u}_{1} \in$ $\mathcal{R}(T)$ such that $\mathcal{R}(f)(\boldsymbol{v}) \leq \boldsymbol{u}_{0} \vee \boldsymbol{u}_{1}$, there are $\boldsymbol{x}_{0}, \boldsymbol{x}_{1} \in \mathcal{R}(S)$ and $\boldsymbol{y} \in S$ such that

$$
f(\boldsymbol{y}) \leq \boldsymbol{u}_{0} \vee \boldsymbol{u}_{1}, \quad \mathcal{R}(f)\left(\boldsymbol{x}_{i}\right) \leq \boldsymbol{u}_{i} \quad \text { for all } i<2, \quad \boldsymbol{v} \leq \boldsymbol{x}_{0} \vee \boldsymbol{x}_{1} \vee \boldsymbol{y} .
$$

For all $i<2$ define

$$
\boldsymbol{x}_{i}=\left\{(\boldsymbol{a}, \boldsymbol{b}, \boldsymbol{c}) \in \boldsymbol{v} \mid(f(\boldsymbol{a}), f(\boldsymbol{b}), f(\boldsymbol{c})) \in \boldsymbol{u}_{i}^{*}\right\} \cup\{(0,0,0)\},
$$

and observe that $\boldsymbol{x}_{0}, \boldsymbol{x}_{1}$, as subsets of $\boldsymbol{v}^{*} \cup\{(0,0,0)\}$, are reduced, that is, $\boldsymbol{x}_{0}, \boldsymbol{x}_{1} \in \mathcal{R}(S)$. It follows from (4.3) that $\mathcal{R}(f)\left(\boldsymbol{x}_{i}\right) \leq \boldsymbol{u}_{i}$ for all $i<2$. An easy application of [17, Lemma 3.1] yields $\left(\boldsymbol{u}_{0} \vee \boldsymbol{u}_{1}\right)^{*} \subseteq \boldsymbol{u}_{0}^{*} \cup \boldsymbol{u}_{1}^{*}$, and so $\bowtie_{S}(\boldsymbol{a}, \boldsymbol{b}, \boldsymbol{c}) \leq \boldsymbol{x}_{0} \vee \boldsymbol{x}_{1}$ for every $(\boldsymbol{a}, \boldsymbol{b}, \boldsymbol{c}) \in \boldsymbol{v}$ such that $(f(\boldsymbol{a}), f(\boldsymbol{b}), f(\boldsymbol{c})) \in$ $\left(\boldsymbol{u}_{0} \vee \boldsymbol{u}_{1}\right)^{*}$.

For all $(\boldsymbol{a}, \boldsymbol{b}, \boldsymbol{c}) \in \boldsymbol{v}$ define

$$
\varrho((\boldsymbol{a}, \boldsymbol{b}, \boldsymbol{c}))= \begin{cases}\boldsymbol{a} & \text { if } f(\boldsymbol{a}) \leq \pi\left(\boldsymbol{u}_{0} \vee \boldsymbol{u}_{1}\right), \\ \boldsymbol{c} & \text { otherwise }\end{cases}
$$

and put

$$
\boldsymbol{y}=\bigvee\left(\varrho((\boldsymbol{a}, \boldsymbol{b}, \boldsymbol{c})) \mid(\boldsymbol{a}, \boldsymbol{b}, \boldsymbol{c}) \in \boldsymbol{v} \text { and }(f(\boldsymbol{a}), f(\boldsymbol{b}), f(\boldsymbol{c})) \notin\left(\boldsymbol{u}_{0} \vee \boldsymbol{u}_{1}\right)^{*}\right)
$$

Clearly, $\boldsymbol{y} \in S$, and, by (4.1), $\bowtie_{S}(\boldsymbol{a}, \boldsymbol{b}, \boldsymbol{c}) \leq \boldsymbol{y}$ for all $(\boldsymbol{a}, \boldsymbol{b}, \boldsymbol{c}) \in \boldsymbol{v}$ such that $(f(\boldsymbol{a}), f(\boldsymbol{b}), f(\boldsymbol{c})) \notin\left(\boldsymbol{u}_{0} \vee \boldsymbol{u}_{1}\right)^{*}$. Overall, we have proved that $\bowtie_{S}(\boldsymbol{a}, \boldsymbol{b}, \boldsymbol{c}) \leq$ $\boldsymbol{x}_{0} \vee \boldsymbol{x}_{1} \vee \boldsymbol{y}$ for all $(\boldsymbol{a}, \boldsymbol{b}, \boldsymbol{c}) \in \boldsymbol{v}$, and so, by (4.2), $\boldsymbol{v} \leq \boldsymbol{x}_{0} \vee \boldsymbol{x}_{1} \vee \boldsymbol{y}$. 
Finally, since $\mathcal{R}(f)(\boldsymbol{v}) \leq \boldsymbol{u}_{0} \vee \boldsymbol{u}_{1}$, it follows from (4.1) that $f(\varrho((\boldsymbol{a}, \boldsymbol{b}, \boldsymbol{c})))$ $\leq \pi\left(\boldsymbol{u}_{0} \vee \boldsymbol{u}_{1}\right)$ for every $(\boldsymbol{a}, \boldsymbol{b}, \boldsymbol{c}) \in \boldsymbol{v}$ such that $(f(\boldsymbol{a}), f(\boldsymbol{b}), f(\boldsymbol{c})) \notin\left(\boldsymbol{u}_{0} \vee \boldsymbol{u}_{1}\right)^{*}$, whence $f(\boldsymbol{y}) \leq \boldsymbol{u}_{0} \vee \boldsymbol{u}_{1}$.

Observe that $\mathcal{R}(S)$ is distributive "relative to" the $(\vee, 0)$-semilattice $S$, that is, for any $\boldsymbol{a}, \boldsymbol{b}, \boldsymbol{c} \in S$ with $\boldsymbol{c} \leq \boldsymbol{a} \vee \boldsymbol{b}$, there are $\boldsymbol{a}^{\prime} \leq \boldsymbol{a}, \boldsymbol{b}^{\prime} \leq \boldsymbol{b}$ in $\mathcal{R}(S)$ such that $\boldsymbol{c}=\boldsymbol{a}^{\prime} \vee \boldsymbol{b}^{\prime}$. It follows that the $(\vee, 0)$-semilattice $\mathcal{R}^{\infty}(S)$ is distributive. By Lemma 3.2, we obtain

Corollary 4.2. The functor $\mathcal{R}^{\infty}$ is diluting. Moreover, $\mathcal{R}^{\infty}(S)$ is a distributive $(\vee, 0)$-semilattice for every $(\vee, 0)$-semilattice $S$.

Observe that the functor $\mathcal{R}^{\infty}$ is denoted by $\mathcal{D}$ in [17].

5. Free trees. Let $k$ be a positive integer and let $\Omega$ be a set. Given a map $\Psi:[\Omega]^{k-1} \rightarrow[\Omega]^{<\omega}$, we say that a $k$-element subset $B$ of $\Omega$ is free (with respect to $\Psi)$ if $b \notin \Psi(B \backslash\{b\})$ for all $b \in B$.

Kuratowski's Free Set Theorem [7]. Let $k$ be a positive integer, let $\Omega$ be a set, and let $\Psi:[\Omega]^{k-1} \rightarrow[\Omega]^{<\omega}$ be any map. If $|\Omega| \geq \aleph_{k-1}$, then there is a k-element free subset of $\Omega$.

Notation. Let $k$ and $n$ be natural numbers with $k>0$. Given a natural number $m \leq n$ and a map $g:\{m, \ldots, n-1\} \rightarrow k$, we shall put

$$
T_{n, k}(g)=\{f: n \rightarrow k \mid f \text { extends } g\}
$$

Given a natural number $m<n$, a map $g:\{m+1, \ldots, n-1\} \rightarrow k$, and $i<k$, we shall use the notation

$$
\begin{aligned}
T_{n, k}(g, i) & =\left\{f \in T_{n, k}(g) \mid f(m)=i\right\}, \\
T_{n, k}(g, \neg i) & =\left\{f \in T_{n, k}(g) \mid f(m) \neq i\right\} .
\end{aligned}
$$

Definition 2. Let $\Omega$ be a set and let $\Phi:[\Omega]^{<\omega} \rightarrow[\Omega]^{<\omega}$ be a map. Let $k$ and $n$ be natural numbers with $k>0$. We say that a family $\mathcal{T}=(\alpha(f) \mid$ $f: n \rightarrow k$ ) of distinct elements of $\Omega$ is a free $k$-tree of height $n$ (with respect to $\Phi)$ if

$$
\left\{\alpha(f) \mid f \in T_{n, k}(g, i)\right\} \cap \Phi\left(\left\{\alpha(f) \mid f \in T_{n, k}(g, \neg i)\right\}\right)=\emptyset
$$

for all $m<n$, all maps $g:\{m+1, \ldots, n-1\} \rightarrow k$, and all $i<k$. We will call the set $\operatorname{rng} \mathcal{T}=\{\alpha(f) \mid f: n \rightarrow k\}$ the range of $\mathcal{T}$.

LEMMA 5.1. Let $\Omega$ be a set and let $\Phi:[\Omega]^{<\omega} \rightarrow[\Omega]^{<\omega}$ be a map. Let $k$ be a positive integer. Every subset $X$ of $\Omega$ of cardinality at least $\aleph_{k-1}$ contains the range of a free $k$-tree of height $n$, for every natural number $n$.

Proof. We shall argue by induction on $n$. If $n=0$, pick $\mathcal{T}=\left\{\alpha_{\emptyset}\right\}$, where $\alpha_{\emptyset}$ is an arbitrary element of $X$. Let $n$ be natural number and suppose that the statement holds for $n$. We shall prove that $X$ contains a free $k$-tree, $\mathcal{T}$, 
of height $n+1$. Decompose $X$ into a union of pairwise disjoint subsets $X_{\xi}$ for $\xi<\omega_{k-1}$ of cardinality at least $\aleph_{k-1}$. By the induction hypothesis, each $X_{\xi}$ contains the range of a free tree $\mathcal{T}_{\xi}=\left(\alpha_{\xi}(f) \mid f: n \rightarrow k\right)$ of height $n$. Define a map $\Psi:\left[\omega_{k-1}\right]^{k-1} \rightarrow\left[\omega_{k-1}\right]^{<\omega}$ by

$$
\Psi(F)=\left\{\nu<\omega_{k-1} \mid \operatorname{rng} \mathcal{T}_{\nu} \cap \Phi\left(\bigcup_{\xi \in F} \operatorname{rng} \mathcal{T}_{\xi}\right) \neq \emptyset\right\}
$$

for all $F \in\left[\omega_{k-1}\right]^{k-1}$ (since the sets $\operatorname{rng} \mathcal{T}_{\xi}$ are pairwise disjoint and finite, $\Psi(F)$ is finite for every $\left.F \in\left[\omega_{k-1}\right]^{k-1}\right)$. By Kuratowski's Free Set Theorem, there is a $k$-element free subset, $B=\left\{\xi_{0}, \ldots, \xi_{k-1}\right\}$, of $X$ with respect to $\Psi$. Put $\alpha(f)=\alpha_{\xi_{f(n)}}(f\lceil n)$ for all maps $f:(n+1) \rightarrow k$. We claim that $\mathcal{T}=(\alpha(f) \mid f:(n+1) \rightarrow k)$ is a free $k$-tree with respect to $\Phi$. Let $m<n+1$ and fix a map $g:\{m+1, \ldots, n\} \rightarrow k$. If $m=n$, the only possibility is $g=\emptyset$. Then

$$
\begin{aligned}
\left\{\alpha(f) \mid f \in T_{n+1, k}(g, i)\right\} & =\operatorname{rng} \mathcal{T}_{\xi_{i}}, \\
\left\{\alpha(f) \mid f \in T_{n+1, k}(g, \neg i)\right\} & =\bigcup_{j<k, j \neq i} \operatorname{rng} \mathcal{T}_{\xi_{j}},
\end{aligned}
$$

for all $i<k$. Since $B$ is a free set with respect to $\Psi$,

$$
\operatorname{rng} \mathcal{T}_{\xi_{i}} \cap \Phi\left(\bigcup_{j<k, j \neq i} \operatorname{rng} \mathcal{T}_{\xi_{j}}\right)=\emptyset,
$$

by (5.2). Let $m<n$ and $i<k$. Put $g^{\prime}=g\lceil\{m+1, \ldots, n-1\}$. Then

$$
\begin{gathered}
\left\{\alpha(f) \mid f \in T_{n+1, k}(g, i)\right\}=\left\{\alpha_{\xi_{g(n)}}(f) \mid f \in T_{n, k}\left(g^{\prime}, i\right)\right\}, \\
\left\{\alpha(f) \mid f \in T_{n+1, k}(g, \neg i)\right\}=\left\{\alpha_{\xi_{g(n)}}(f) \mid f \in T_{n, k}\left(g^{\prime}, \neg i\right)\right\} .
\end{gathered}
$$

Since $\mathcal{T}_{\alpha_{\xi_{g(n)}}}$ is a free $k$-tree with respect to $\Phi$,

$$
\left\{\alpha_{\xi_{g(n)}}(f) \mid f \in T_{n, k}\left(g^{\prime}, i\right)\right\} \cap \Phi\left(\left\{\alpha_{\xi_{g(n)}}(f) \mid f \in T_{n, k}\left(g^{\prime}, \neg i\right)\right\}\right)=\emptyset,
$$

by (5.1).

6. The optimal bound in Wehrung's theorem. Let $\mathcal{F}$ be an expanding functor on $\mathbf{S}$ satisfying the following properties: For every $(\vee, 0)$-semilattice $S$ and every family $\left(S_{i} \mid i \in I\right)$ of $(\vee, 0)$-subsemilattices of $S$,

$$
\bigcap_{i \in I} \mathcal{F}\left(S_{i}\right)=\mathcal{F}\left(\bigcap_{i \in I} S_{i}\right) \text {. }
$$

For a nonempty upwards directed poset $P$ and every family $\left(S_{p} \mid p \in P\right)$ of $(\vee, 0)$-semilattices such that $S_{p}$ is a $(\vee, 0)$-subsemilattice of $S_{q}$ whenever $p \leq q$ in $P$,

$$
\bigcup_{p \in P} \mathcal{F}\left(S_{p}\right)=\mathcal{F}\left(\bigcup_{p \in P} S_{p}\right)
$$


Put $\mathcal{G}=\mathcal{F} \circ \mathcal{L}$. Then for every set $\Omega$ and every family $\left(A_{i} \mid i \in I\right)$ of subsets of $\Omega$,

$$
\bigcap_{i \in I} \mathcal{G}\left(A_{i}\right)=\mathcal{G}\left(\bigcap_{i \in I} A_{i}\right)
$$

and for a nonempty upwards directed poset $P$ and every family $\left(A_{p} \mid p \in P\right)$ of sets such that $A_{p} \subseteq A_{q}$ whenever $p \leq q$ in $P$,

$$
\bigcup_{p \in P} \mathcal{G}\left(A_{p}\right)=\mathcal{G}\left(\bigcup_{p \in P} A_{p}\right) \text {. }
$$

It follows that, given a set $\Omega$ and an element $\boldsymbol{a} \in \mathcal{G}(\Omega)$, there is a smallest finite subset $F$ of $\Omega$ such that $\boldsymbol{a} \in \mathcal{G}(F)$. We shall call the subset $F$ the support of $\boldsymbol{a}$. We denote the support of $\boldsymbol{a}$ by $\operatorname{Supp}(\boldsymbol{a})$ (see [17]). Now we rephrase [17, Theorem 6.1].

THEOREM 6.1. Let $\Omega$ be a set of cardinality at least $\aleph_{2}$ and let $\mathcal{F}$ be a diluting functor satisfying (6.1) and (6.2). Let $A$ be an algebra with either a congruence-compatible structure of a $(\vee, 1)$-semilattice or a congruence compatible structure of a lattice. Then there does not exist a weakly distributive $(\vee, 0)$-homomorphism from $\operatorname{Con}_{\mathrm{c}} A$ to $\mathcal{G}(\Omega)$ containing 1 in its range.

Proof. Assume for contradiction that there exists a weakly distributive $(\vee, 0)$-homomorphism $\mu$ : $\operatorname{Con}_{\mathrm{c}} A \rightarrow \mathcal{G}(\Omega)$ with 1 in its range. Since 1 is in the range of $\mu$, there is a finite subset $T$ of $\Omega$ such that

$$
\mu\left(\bigvee\left\{\Theta_{A}(s, t) \mid s, t \in T\right\}\right)=1
$$

Put $s=1$ (the largest element of $A$ ) if $A$ has a congruence-compatible structure of a $(\vee, 1)$-semilattice, and $s=\bigvee T$ if $A$ has a congruence compatible structure of a lattice. Then

$$
\mu\left(\bigvee_{t \in T} \Theta_{A}(s, t)\right)=\bigvee_{t \in T} \mu\left(\Theta_{A}(s, t)\right)=1
$$

Let $\xi \in \Omega$. Since the homomorphism $\mu$ is weakly distributive, there are $\theta_{0}^{\xi}, \theta_{1}^{\xi} \in \operatorname{Con}_{\mathrm{c}} A$ such that $\bigvee_{t \in T} \Theta_{A}(s, t) \leq \theta_{0}^{\xi} \vee \theta_{1}^{\xi}$ and $\mu\left(\theta_{i}^{\xi}\right) \leq \boldsymbol{a}_{i}^{\xi}$ for $i=0,1$. In particular, $\Theta_{A}(s, t) \leq \theta_{0}^{\xi} \vee \theta_{1}^{\xi}$ for every $t \in T$. Fix $t \in T$. Since $\Theta_{A}(s, t) \leq$ $\theta_{0}^{\xi} \vee \theta_{1}^{\xi}$, there are a positive integer $n_{\xi}$ and elements $t=z_{0}^{\xi}, z_{1}^{\xi}, \ldots, z_{n_{\xi}}^{\xi}=s$ in $A$ such that

$$
\mu \Theta_{A}\left(z_{i}^{\xi}, z_{i+1}^{\xi}\right) \leq \boldsymbol{a}_{\varepsilon(i)}^{\xi} \quad \text { for all } i<n_{\xi},
$$

for all $\xi \in \Omega$. (Recall that $\varepsilon(i)=i \bmod 2$.)

If $A$ has a congruence-compatible structure of a $(\vee, 1)$-semilattice, we replace each $z_{i}^{\xi}$ with $\bigvee_{j=0}^{i} z_{j}^{\xi}$. If $A$ has a congruence-compatible structure of a lattice, we replace each $z_{i}^{\xi}$ with $s \wedge\left(\bigvee_{j=0}^{i} z_{j}^{\xi}\right)$. In both cases we obtain an 
increasing chain $t=z_{0}^{\xi} \leq z_{1}^{\xi} \leq \cdots \leq z_{n_{\xi}}^{\xi}=s$ in $A$ such that (6.3) remains satisfied.

As in [17, Section 6], we denote by $\operatorname{Con}_{\mathrm{c}}^{U} A$ the $(\vee, 0)$-subsemilattice of $\operatorname{Con}_{\mathrm{c}} A$ generated by all principal congruences $\Theta_{A}(u, v)$, where $u, v \in U$, for every subset $U$ of $A$. Further, we denote by $S(F)$ the $(\vee, 0)$-subsemilattice of $A$ generated by $\left\{z_{i}^{\xi} \mid \xi \in F\right.$ and $\left.0 \leq i \leq n_{\xi}\right\}$, and we put

$$
\Phi(F)=\bigcup\left\{\operatorname{Supp}(\mu \theta) \mid \theta \in \operatorname{Con}_{\mathrm{c}}^{S(F)} A\right\}
$$

for every subset $F$ of $\Omega$. Observe that if the subset $F$ is finite, then both $S(F)$ and $\Phi(F)$ are finite.

Since the size of $\Omega$ is at least $\aleph_{2}$, there are a positive integer $n$ and a subset $X$ of $\Omega$ of cardinality at least $\aleph_{2}$ such that $n_{\xi}=n$ for all $\xi \in X$. The following crucial claim is analogous to [17, Lemma 6.2], giving another illustration of the "erosion method".

Claim 1. Let $\mathcal{T}=(\alpha(f) \mid f: n \rightarrow 3)$ be a free 3 -tree with respect to $\Phi$ with $\operatorname{rng} \mathcal{T} \subseteq X$. Then

$$
\Theta_{A}\left(s, \bigvee_{f \in T_{n, 2}(g)} z_{n-m}^{\alpha(f)}\right)=0
$$

for every nonnegative integer $m \leq n$ and every map $g:\{m, \ldots, n-1\} \rightarrow 2$.

Proof of Claim. We shall argue by induction on $m$. If $m=0$, then the equality (6.5) is trivially satisfied for every map $g:\{m, \ldots, n-1\} \rightarrow 2$. Let $m<n$, let $g:\{m+1, \ldots, n-1\} \rightarrow 2$ be a map, and suppose that (6.5) is satisfied at stage $m$. Put

$$
x_{i}=\bigvee_{f \in T_{n, 2}(g, i)} z_{n-m-1}^{\alpha(f)} \quad \text { for all } i<2 .
$$

Fix $i<2$. Clearly,

$$
\mu \Theta_{A}\left(s, x_{i}\right) \leq\left(\bigvee_{f \in T_{n, 2}(g, i)} \mu \Theta_{A}\left(z_{n-m-1}^{\alpha(f)}, z_{n-m}^{\alpha(f)}\right)\right) \vee \mu \Theta_{A}\left(s, \bigvee_{f \in T_{n, 2}(g, i)} z_{n-m}^{\alpha(f)}\right)
$$

Put

$$
\boldsymbol{v}=\mu \Theta_{A}\left(s, \bigvee_{f \in T_{n, 2}(g)} z_{n-m-1}^{\alpha(f)}\right)=\mu \Theta_{A}\left(s, x_{0} \vee x_{1}\right)
$$

Put $A_{i}=\left\{\alpha(f) \mid f \in T_{n, 2}(g, i)\right\}$, and let $\varphi_{i}: A_{i} \rightarrow 2$ be the constant map with the value $\varepsilon(n-m-1)$. By the induction hypothesis

$$
\mu \Theta_{A}\left(s, \bigvee_{f \in T_{n, 2}(g, i)} z_{n-m}^{\alpha(f)}\right)=0
$$

and, by (6.3), $\mu \Theta_{A}\left(z_{n-m-1}^{\alpha(f)}, z_{n-m}^{\alpha(f)}\right) \leq \boldsymbol{a}_{\varepsilon(n-m-1)}^{\alpha(f)}$ for all $f \in T_{n, 2}(g, i)$. Thus 


$$
\mu \Theta_{A}\left(s, x_{i}\right) \leq \bigvee_{f \in T_{n, 2}(g, i)} \boldsymbol{a}_{\varepsilon(n-m-1)}^{\alpha(f)}=\boldsymbol{a}_{\varphi_{i}}^{A_{i}} .
$$

Let $\delta$ be any element of $X$. By the Erosion Lemma [17, Lemma 5.1], there are $\boldsymbol{u}_{j} \in \operatorname{Con}_{c}^{S\left(A_{j} \cup\{\delta\}\right)} A$ such that $\boldsymbol{v} \leq \boldsymbol{u}_{0} \vee \boldsymbol{u}_{1}$ and $\boldsymbol{u}_{j} \leq \boldsymbol{a}_{\varepsilon(j)}^{\delta}, \mu \Theta_{A}\left(s, x_{j}\right)$ (and so $\boldsymbol{u}_{j} \leq \boldsymbol{a}_{\varepsilon(j)}^{\delta}, \boldsymbol{a}_{\varphi_{j}}^{A_{j}}$ ) for all $j<2$.

Now let $\delta=\alpha(f)$ for some $f \in T_{n, 3}(g, 2)$. By (6.4), $\operatorname{Supp}(\boldsymbol{v}) \subseteq \Phi(\{\alpha(f) \mid$ $\left.\left.f \in T_{n, 2}(g)\right\}\right)=\Phi\left(A_{0} \cup A_{1}\right)$ and $\operatorname{Supp}\left(\boldsymbol{u}_{j}\right) \subseteq \Phi\left(A_{j} \cup\{\delta\}\right)$ for all $j<2$. Since $\mathcal{T}$ is a free 3 -tree with respect to $\Phi$, we have $\delta \notin \Phi\left(A_{0} \cup A_{1}\right)$ and $A_{1-j} \cap \Phi\left(A_{j} \cup\{\delta\}\right)=\emptyset$ for all $j<2$. It follows that $\boldsymbol{v} \in \mathcal{G}(\Omega \backslash\{\delta\})$ and $\boldsymbol{u}_{j} \in \mathcal{G}\left(\Omega \backslash A_{1-j}\right)$ for all $j<2$. Since the functor $\mathcal{F}$ is diluting, applying Lemma 3.3, we conclude that $\boldsymbol{v}=0$ as desired. Claim 1

Now we finish the proof of Theorem 6.1. By Lemma 5.1, the set $X$ contains the range of a free 3-tree $\mathcal{T}=(\alpha(f) \mid f: n \rightarrow 3)$ of height $n$. By Claim 1,

$$
\Theta_{A}\left(s, \bigvee_{f \in T_{n, 2}(g)} z_{n-m}^{\alpha(f)}\right)=0
$$

for every nonnegative integer $m \leq n$ and every map $g:\{m, \ldots, n-1\} \rightarrow 2$. For $m=n$ and $g=\emptyset$ we have

$$
\bigvee_{f \in T_{n, 2}(g)} z_{n-m}^{\alpha(f)}=\bigvee_{f: n \rightarrow 2} z_{0}^{\alpha(f)}=t,
$$

whence $\Theta_{A}(s, t)=0$ for every $t \in T$. Consequently, $\bigvee_{t \in T} \Theta_{A}(s, t)=0$, which leads to a contradiction.

By Corollary 4.2, the functor $\mathcal{R}^{\infty}$ is diluting, and by [17, Lemma 3.6], it satisfies both (6.1) and (6.2). Let us denote by $\mathcal{G}$ the composition $\mathcal{R}^{\infty} \circ \mathcal{L}$ (it is the same $\mathcal{G}$ as the one considered in [17]). Since $\mathcal{R}^{\infty}(S)$ is distributive for every $(\vee, 0)$-semilattice $S$, we obtain the following corollary.

COROLlary 6.2. Let $\Omega$ be a set of cardinality at most $\aleph_{2}$. Then there is no lattice $L$ such that $\mathcal{G}(\Omega)$ is isomorphic to $\operatorname{Con}_{\mathrm{c}} L$.

A. P. Huhn [6] (see also [3, Theorem 13 in Appendix C]) proved that every distributive $(\vee, 0)$-semilattice of size at most $\aleph_{1}$ is isomorphic to $\operatorname{Con}_{\mathrm{c}} L$ for some lattice $L$. Moreover, the lattice $L$ can be taken sectionally complemented and modular [16, Corollary 5.3], or relatively complemented, locally finite, and with zero [4]. In particular, in all these cases, the lattice $L$ has permutable congruences [1].

Acknowledgments. I thank Miroslav Ploščica, Philippe Toffin, Jiří Tůma, and Friedrich Wehrung for their support during my visit to the University of Caen. Special thanks are due to Friedrich Wehrung who carefully 
read the manuscript of the paper. His suggestions considerably improved its final version.

\section{References}

[1] R. P. Dilworth, The structure of relatively complemented lattices, Ann. of Math. (2) 51 (1950), 348-359.

[2] N. Funayama and T. Nakayama, On the distributivity of a lattice of lattice-congruences, Proc. Imp. Acad. Tokyo 18 (1942), 553-554.

[3] G. Grätzer, General Lattice Theory, 2nd ed., Birkhäuser, Basel, 1998.

[4] G. Grätzer, H. Lakser, and F. Wehrung, Congruence amalgamation of lattices, Acta Sci. Math. (Szeged) 66 (2000), 339-358.

[5] G. Grätzer and E. T. Schmidt, On congruence lattices of lattices, Acta Math. Acad. Sci. Hungar. 13 (1962), 179-185.

[6] A. P. Huhn, On the representation of distributive algebraic lattices I-III, Acta Sci. Math. (Szeged) 45 (1983), 139-246; 53 (1989), 3-10 and 11-18.

[7] C. Kuratowski, Sur une caractérisation des alephs, Fund. Math. 38 (1951), 14-17.

[8] M. Ploščica, Congruence lattices of lattices with m-permutable congruences, preprint, 2007.

[9] M. Ploščica and J. Tůma, Uniform refinements in distributive semilattices, in: Contributions to General Algebra 10 (Klagenfurt, 1997), Verlag Johannes Heyn, Klagenfurt, 1998.

[10] M. Ploščica, J. Tůma, and F. Wehrung, Congruence lattices of free lattices in nondistributive varieties, Colloq. Math. 76 (1998), 269-278.

[11] P. Růžička, J. Tůma, and F. Wehrung, Distributive congruence lattices of congruence-permutable algebras, J. Algebra 311 (2007), 96-116.

[12] J. Tůma and F. Wehrung, A survey on recent results on congruence lattices of lattices, Algebra Universalis 45 (2002), 439-471.

[13] -, -, Simultaneous representations of semilattices by lattices with permutable congruences, Int. J. Algebra Comput. 11 (2001), 217-246.

[14] F. Wehrung, Non-measurability properties of interpolation vector spaces, Israel J. Math. 103 (1998), 177-206.

[15] -, A uniform refinement property for congruence lattices, Proc. Amer. Math. Soc. 127 (1999), 363-370.

[16] - Representation of algebraic distributive lattices with $\aleph_{1}$ compact elements as ideal lattices of regular rings, Publ. Mat. (Barcelona) 44 (2000), 419-435.

[17] - A solution to Dilworth's congruence lattice problem, Adv. Math. 216 (2007), $610-625$.

Department of Algebra

Faculty of Mathematics and Physics

Charles University

Sokolovská 83

18675 Praha 8, Czech Republic

E-mail: ruzicka@karlin.mff.cuni.cz 